\title{
A retrospective analysis of 1,231 patients with anemia after surgical treatment of hyperthyroidism
}

\author{
RUISHA TU ${ }^{1}$, HONGFENG SHEN ${ }^{2}$, QICONG ZHU ${ }^{3}$, GAOCAI MAO $^{4}$, XIAOJIANG XU $^{1}$ and SHUYONG YU ${ }^{1}$ \\ Departments of ${ }^{1}$ Neck and Thoracic Surgery, ${ }^{2}$ Urinary Surgery, ${ }^{3}$ Internal Medicine-Oncology and \\ ${ }^{4}$ Pathology, 187th Hospital of People's Liberation Army, Haikou, Hainan 571100, P.R. China
}

Received March 9, 2018; Accepted August 28, 2018

DOI: $10.3892 /$ etm.2018.6738

\begin{abstract}
This study retrospectively analyzed the clinical and pathological data of 1,231 patients affected by anemia after surgical treatment of hyperthyroidism to explore the influencing factors of anemia after surgical treatment of hyperthyroidism. The clinical data of 1,231 patients affected by anemia after surgical treatment of hyperthyroidism from 1987 to 2017 were analyzed. Clinical data included the surgery methods, sex, age and pathological types. SPSS 22.0 statistical software was used for all statistical analyses. Correlation analyses were performed by using logistic regression analysis, and other enumeration data were subjected to $\chi^{2}$ test. $\mathrm{p}<0.05$ was considered to be statistically significant. The occurrence of anemia after surgical treatment of hyperthyroidism was significantly correlated with age and pathological types $(\mathrm{p}<0.05)$. Correlation analysis also showed that age and pathological types were significantly correlated with the occurrence of anemia after surgical treatment of hyperthyroidism. Age and pathological types may be the risk factors for anemia in patients with surgical treatment of hyperthyroidism. Age and pathological type were significantly correlated with the occurrence of anemia after surgical treatment of hyperthyroidism, and may be risk factors for this disease.
\end{abstract}

\section{Introduction}

Hyperthyroidism is a systemic metabolic disorder caused by excessive synthesis and secretion of the thyroid hormone $(1,2)$. Hyperthyroidism at an early stage may show bone and joint pain, vomiting, hypercalcemia and other symptoms (3). With the changes in people's lifestyle and increased psychological pressure, the incidence of hyperthyroidism is also significantly

Correspondence to: Dr Shuyong Yu, Department of Neck and Thoracic Surgery, 187th Hospital of People's Liberation Army, 100 Longkun South Road, Longhua, Haikou, Hainan 571100, P.R. China

E-mail: yhy7au@163.com

Key words: hyperthyroidism, retrospective analysis, surgery, postoperative anemia increasing. Studies have shown hyperthyroidism affects 31 in 100,000 people, and $60 \%$ of the patients had a family orientation. The incidence of hyperthyroidism is higher in women than in men (1:4-6). The incidence of hyperthyroid is high in China, and studies have shown that the incidence of hyperthyroidism in China is up to $3 \%$, seriously affecting patients' work, life and learning ability. Surgical treatment is the main treatment for hyperthyroidism. Studies have shown that the efficacy of surgical treatment of hyperthyroidism is better than that of drug treatment. However, surgical treatment of hyperthyroidism may cause some complications, of which anemia is a major complication, and severe anemia may even affect patients' cardiopulmonary function, leading to poor postoperative recovery $(2,4,5)$. Therefore, effective prevention and treatment of anemia is an important guarantee for the success of surgical treatment of patients with hyperthyroidism. In this study, clinical data of 1,231 cases of hyperthyroidism combined with moderate anemia were collected and retrospectively analyzed to investigate the influencing factors of anemia after surgical treatment of hyperthyroidism, so as to provide guidance for the prevention of postoperative anemia in hyperthyroidism patients.

\section{Patients and methods}

Research subjects. A total of 1,231 hyperthyroidism patients combined with postoperative anemia were enrolled in 187th Hospital of People's Liberation Army (Haikou, China). Those patients included 658 female patients (53.5\%) and 573 male patients $(46.5 \%)$, and the average age was $35.4 \pm 3.4$ years. There were 456 cases of Graves' disease, accounting for $37.0 \%$, 408 cases of multi-nodular goiter with hyperthyroidism, accounting for $33.1 \%$, and 367 cases of thyroid cancer with hyperthyroidism, accounting for $29.9 \%$. During surgery, 418 patients were subjected to subtotal resection of both sides; 405 patients were subjected to subtotal resection of one side and total resection of the other side, and 413 patients were subjected to total resection of both sides.

\section{Inclusion and exclusion criteria}

Inclusion criteria. Hyperthyroidism patients who met the surgical indications were enrolled in the study. Diagnostic criteria for hyperthyroidism or clinical manifestations of patients with hyperthyroidism were: Hyperphagia, intolerance 
Table I. Comparison of incidence of postoperative anemia among patients with different clinical parameters.

\begin{tabular}{|c|c|c|c|c|c|}
\hline Items & Cases & $\begin{array}{c}\text { Cases of } \\
\text { postoperative } \\
\text { anemia }\end{array}$ & $\begin{array}{c}\text { Postoperative } \\
\text { anemia rate }(\%)\end{array}$ & $\begin{array}{l}\text { Statistical } \\
\text { value }\end{array}$ & P-value \\
\hline Sex & & & & 0.040 & 0.840 \\
\hline Male & $573(46.5)$ & 83 & 14.5 & & \\
\hline Female & $658(53.5)$ & 98 & 14.9 & & \\
\hline Age (years) & & & & 4.405 & 0.035 \\
\hline$>30$ & $635(51.6)$ & 120 & 18.9 & & \\
\hline$\leq 30$ & $596(48.4)$ & 86 & 14.4 & & \\
\hline Surgical approach & & & & 0.471 & 0.789 \\
\hline Subtotal resection & $418(34.0)$ & 56 & 13.4 & & \\
\hline Total/subtotal resection & $405(32.5)$ & 59 & 14.6 & & \\
\hline Total resection & $413(33.5)$ & 62 & 15.0 & & \\
\hline Pathological types & & & & 0.031 & 0.969 \\
\hline Graves' disease & $456(37.0)$ & 69 & 15.1 & & \\
\hline Multi-nodular hyperthyroidism ${ }^{a}$ & $408(33.1)$ & 88 & 21.5 & & \\
\hline Hyperthyroidism and cancer ${ }^{a, b}$ & 367 (29.9) & 90 & 24.5 & & \\
\hline
\end{tabular}

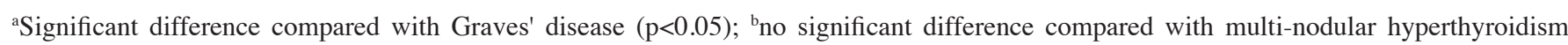
$(\mathrm{p}<0.05)$.

of heat, palpitations, agitation and other high metabolic syndrome, exophthalmos and varying degrees of goiter (6-8), laboratory tests show high levels of free triiodothyronine FT3 or free thyroid hormone FT4, decreased thyroid hormone TSH levels and anti-thyroid antibody-positive. Indications for surgical treatment for hyperthyroidism: Long-term use of drugs with poor results; secondary hyperthyroidism caused by other factors, moderate or beyond primary hyperthyroidism; hyperthyroidism with oppression; recurrence after drug treatment; bone marrow suppression and other toxic response.

The patients or their relatives signed informed consent, and this study was approved by the Ethics Committee of 187th Hospital of People's Liberation Army.

Exclusion criteria. Patients with hypothyroidism; patients who could not tolerate surgery; patients with coagulopathy and clotting factor deficiency; patients with heart and kidney dysfunction; patients with cerebrovascular accident; patients with serious complications; patients allergic to anesthetics; patients with preoperative liver disease, kidney diseases, gastrointestinal bleeding, cancer and other diseases caused by anemia.

\section{Research methods}

Diagnostic criteria of moderate anemia. Anemia: All patients showed a reduction in hemoglobin $(\mathrm{Hb})$ concentration on the day of surgery, anemia was diagnosed at $\mathrm{Hb}$ levels below $120 \mathrm{~g} / \mathrm{l}$ for male, below $<110 \mathrm{~g} / \mathrm{l}$ for female, and below $<110 \mathrm{~g} / 1$ for pregnant women $(9,10)$. Moderate anemia: $\mathrm{Hb} 60-90 \mathrm{~g} / \mathrm{l}$, severe anemia: $\mathrm{Hb} 60-31 \mathrm{~g} / 1$, and severe anemia: $\mathrm{Hb} \leq 30 \mathrm{~g} / \mathrm{l}$.

Surgical methods. Total resection of both sides: Anesthesia was performed for regional nerve block. Two thyroids were fully exposed and the upper and inferior artery, as well as the middle and inferior veins were ligated to separate blood vessels. Dorsal thyroid was exposed and resected. Posterior margin of the thyroid was retained for benign lesions. Parathyroid artery blood supply was maintained, and laryngeal nerve accidental injury and damage to its vascular branches were avoided. Subtotal resection of both sides: Basically, the same as total resection, but glandular tissue and dorsal envelope were retained. Total resection on one side and subtotal resection on the other side: Basically, the same as total resection, but glandular tissue and dorsal envelope of one side were retained.

Statistical analysis. Statistical software SPSS V22.0 (Version X; IBM, Armonk, NY, USA) was used. Normal distribution analysis was performed. Normal distribution data were expressed as mean $\pm \mathrm{SD}$. Correlation analyses were performed by using logistic regression analysis. Comparison among multiple groups was done using one-way ANOVA test followed by post hoc test (Least Significant Difference). F-test and paired t-test were used for the comparison of the incidence of postoperative anemia between the groups. Comparison of rates was performed by using $\chi^{2}$ test. $\mathrm{P}<0.05$ was considered to indicate a statistically significant difference.

\section{Results}

Comparison of incidence of postoperative anemia among patients with different age, sex, surgical methods and pathological types. The incidence of postoperative anemia in female patients was slightly higher than that in male patients, but there was no significant difference $(p>0.05)$. The proportion of anemia patients in hyperthyroidism patients aged $>30$ years was higher than that of patients $\leq 30$ years $(p<0.05)$. F-test and paired t-test showed no significant difference in the incidence 


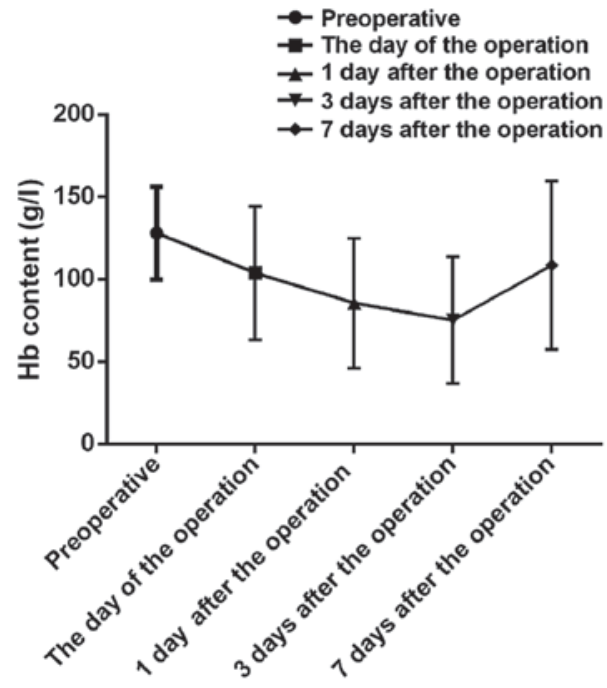

Figure $1 . \mathrm{Hb}$ changes over time. Hb level showed a downward trend from the day of surgery to the 3rd day after surgery, and $\mathrm{Hb}$ increased at the 7th day after surgery. $\mathrm{Hb}$, hemoglobin.

Table II. Changes of $\mathrm{Hb}$ at different stages.

\begin{tabular}{lrrc}
\hline & & \multicolumn{2}{c}{ Hb content $(g / l)$} \\
\cline { 3 - 4 } Stages & Mean \pm SD & Minimum & Maximum \\
\hline Pre-operation & $124.31 \pm 12.52$ & 102 & 158 \\
The day of operation & $102.19 \pm 17.44$ & 64 & 145 \\
One day after operation & $87.65 \pm 15.58$ & 45 & 124 \\
Three days after operation & $74.21 \pm 12.73$ & 37 & 114 \\
Seven days after operation & $98.41 \pm 16.24$ & 63 & 164 \\
\hline
\end{tabular}

$\mathrm{Hb}$, hemoglobin.

of postoperative anemia among patients that received total resection on both sides, subtotal resection on both sides and total resection on one side and subtotal resection on the other side ( $p>0.05)$, indicating that surgical methods have no effect on the incidence of postoperative anemia. F-test showed no significant difference in the incidence of postoperative anemia among patients with Graves' disease, multi-nodular goiter with hyperthyroidism and thyroid cancer with hyperthyroidis $(p>0.05)$, while the paired t-test showed that the proportion of anemia patients in patients with multinodular goiter with hyperthyroidism and thyroid cancer with hyperthyroidis was significantly higher than that of patients with Graves' disease $(\mathrm{p}<0.05)$, while no significant difference in the incidence of postoperative anemia was found between patients with multi-nodular goiter with hyperthyroidism and thyroid cancer with hyperthyroidis $(\mathrm{p}>0.05)$ (Table I).

Changes of $\mathrm{Hb}$ at different stages. All patients presented varying degrees of $\mathrm{Hb}$ decline on the day of surgery. $\mathrm{Hb}$ decreased continuously on the first postoperative day. $\mathrm{Hb}$ decreased to the lowest level $(74.21 \pm 12.53 \mathrm{~g} / \mathrm{l})$ on the $3 \mathrm{rd}$ postoperative day. On the 7th day, $\mathrm{Hb}$ level showed an upward trend (Table II; Fig. 1).
Table III. Correlations of age and pathological types with occurrence of postoperative anemia.

\begin{tabular}{lcc}
\hline Variables & Age (years) & Pathological types \\
\hline$\beta$ & 0.756 & 0.645 \\
t-test & 4.878 & 5.326 \\
P-value & 0.004 & 0.002 \\
\hline
\end{tabular}

Table IV. Univariate Cox regression analysis of factors related to occurrence of postoperative anemia.

\begin{tabular}{lccc}
\hline Factors & P-value & OR & $95 \%$ CI \\
\hline Age (years) & 0.0473 & 1.231 & $(1.121,1.687)$ \\
Pathological types & 0.0458 & 1.657 & $(1.536,2.835)$ \\
\hline
\end{tabular}

OR, odds ratio; CI, confidence interval.

Logistics regression analysis. Correlation analysis showed that age and pathological types were significantly correlated with the occurrence of anemia after the surgical treatment of hyperthyroidism. Age and pathological types may be the risk factors for anemia in patients with surgical treatment of hyperthyroidism (Tables III and IV).

\section{Discussion}

Hyperthyroidism is a systemic metabolic disorder mainly caused by excessive synthesis and secretion of the thyroid hormone. Clinically, hyperthyroidism is usually treated by radiotherapy (11-13) or surgery (14). In this study, clinical data (surgery methods, sex, age and pathological types) of 1,231 patients affected by anemia after surgical treatment of hyperthyroidism from 1987 to 2017 were analyzed.

Retrospective analysis of 1,231 patients with moderate postoperative anemia showed that incidence of postoperative anemia in female patients was slightly higher than that in male patients, but there was no significant difference, which is consistent with the findings reported by Jiang et al (15). Hyperthyroidism may occur in all age groups, but mainly affects patients between 20-40 years. Therefore, patients in this study were divided into two groups of $\leq 30$ years and $>30$ years. In the process of data collection, we found that anemia may occur in patients with different pathological types and different surgical treatments, while surgery methods showed no significant effects on the occurrence of postoperative anemia, but pathological types showed significant effects on the occurrence of postoperative anemia. The causes of anemia after surgical treatment of hyperthyroidism patients are still unclear, and may be related to the following factors: Reduction of thyroid hormone production may lead to reduced erythropoietin, which in turn causes anemia; emergence of erythrocyte-dependent antibodies after surgery, leading to hemolytic anemia; FT3 or FT4, and TSH may be involved in postoperative anemia in patients with hyperthyroidism; autoimmune factors (15-18). M'Rabet-Bensalah et al (19) showed a 
higher prevalence of anemia in patients with hyperthyroidism, but the incidence of anemia was not increased in patients with subclinical thyroid insufficiency, which may be related to the degree of subclinical thyroid dysfunction. The overall level of thyroid dysfunction in these patients does not lead to a pathological change in the level of thyroid stimulating hormone. In this study, correlation analysis showed that age and pathological types, but not surgery methods, were significantly correlated with the occurrence of anemia after surgical treatment of hyperthyroidism. Age and pathological types may be the risk factors for anemia in patients with surgical treatment of hyperthyroidism. Abnormal thyroid function usually caused anemia, and the majority is positive cytochrome anemia.

Iron is the raw material for the synthesis of $\mathrm{Hb}$. Fischli et al (20) showed that abnormalities of iron metabolism in patients with hyperthyroidism could affect $\mathrm{Hb}$ synthesis. Aging can significantly affect body function as well as intake and utilization of iron. This study did not rule out hyperthyroidism patients who were treated with chemotherapy and radiotherapy, which are important risk factors affecting anemia (21). We will solve this problem in our future study.

Advantages of this study were the wide range of age distribution, large sample size, strict inclusion and exclusion criteria as well as wide range of time. These advantages increased the reliability of the results in this study.

In conclusion, there are many possible causes of anemia in patients with hyperthyroidism, and there are few studies on factors related to moderate anemia after surgical treatment of hyperthyroidism. This study retrospectively analyzed 1,231 hyperthyroidism patients who were affected by moderate anemia. We found that age and pathological types, but not sex and surgery methods, were significantly correlated with the occurrence of anemia after surgical treatment of hyperthyroidism. Age and pathological type may be the risk factors for anemia in patients with surgical treatment of hyperthyroidism. The causes and influencing factors of anemia in patients with hyperthyroidism after surgery still need to be further studied $(22,23)$.

\section{Acknowledgements}

Not applicable.

\section{Funding}

No funding was received.

\section{Availability of data and materials}

The datasets used and/or analyzed during the present study are available from the corresponding author on reasonable request.

\section{Authors' contributions}

RT analyzed and interpreted the patient data, and was a major contributor in writing the manuscript. HS performed the experiment and participated in the design of the study. QZ participated in the analysis and discussion of the data. GM was responsible for the collection of the data and the follow-up management of the patients. XX was a major contributor in designing the methods. SY was a major contributor in designing the idea of this study and was responsible for reviewing. All authors have read and approved the final manuscript.

\section{Ethics approval and consent to participate}

This study was approved by the Ethics Committee of 187th Hospital of People's Liberation Army (Haikou, China). Signed informed consents were obtained from the patients or guardians.

\section{Patient consent for publication}

Not applicable.

\section{Competing interests}

The authors declare that they have no competing interests.

\section{References}

1. Krohn T, Hänscheid H, Müller B, Behrendt FF, Heinzel A, Mottaghy FM and Verburg FA: Maximum dose rate is a determinant of hypothyroidism after 131I therapy of Graves' disease but the total thyroid absorbed dose is not. J Clin Endocrinol Metab 99: 4109-4115, 2014.

2. Rosário PW, Batista KC and Calsolari MR: Radioiodine-induced oxidative stress in patients with differentiated thyroid carcinoma and effect of supplementation with vitamins $C$ and $E$ and selenium (antioxidants). Arch Endocrinol Metab 60: 328-332, 2016.

3. Liu J, Tang X, Cheng J, Yang X and Wang Y: Persistent arthralgia, vomiting and hypercalcemia as the initial manifestations of hyperthyroidism: A case report. Mol Clin Oncol 6: 258-260, 2017.

4. Evenson A, Mitchell J, Wei W, Poylin V, Parangi S and Hasselgren PO: The gene expression and activity of calpains and the muscle wasting-associated ubiquitin ligases, atrogin-1 and MuRF1, are not altered in patients with primary hyperparathyroidism. Int J Mol Med 18: 471-475, 2006.

5. Zhang W, Gao R, Yu Y, Guo K, Hou P, Yu M, Liu Y and Yang A: Iodine-131 induces apoptosis in HTori-3 human thyrocyte cell line and G2/M phase arrest in a p53-independent pathway. Mol Med Rep 11: 3148-3154, 2015.

6. Li H, Yuan Y, Zhang Y, He Q, Xu R, Ge F and Wu C: Celastrol inhibits IL- $1 \beta$-induced inflammation in orbital fibroblasts through the suppression of NF- $\kappa \mathrm{B}$ activity. Mol Med Rep 14: 2799-2806, 2016

7. Liu L, Lu H, Liu Y, Liu C and Xun C: Predicting relapse of Graves' disease following treatment with antithyroid drugs. Exp Ther Med 11: 1453-1458, 2016.

8. Reddy PA, Harinarayan CV, Sachan A, Suresh V and Rajagopal G: Bone disease in thyrotoxicosis. Indian J Med Res 135: 277-286, 2012.

9. Roomi MW, Kalinovsky T, Rath M and Niedzwiecki A: Cytokines, inducers and inhibitors modulate MMP-2 and MMP-9 secretion by human Fanconi anemia immortalized fibroblasts. Oncol Rep 37: 1842-1848, 2017.

10. Zhou S, Zhuang Y, Zhao W, Jiang B, Pan H, Zhang X, Peng H and Chen Y: Protective roles of erythropoiesis-stimulating proteins in chronic heart failure with anemia. Exp Ther Med 8: 863-870, 2014.

11. Song X, Meng Z, Jia Q, Zhang L, Xu K, Tan J, Zhang G, Zheng W, Li X and Zhang J: Different radioiodine dose for remnant thyroid ablation in patients with differentiated thyroid cancer: A meta-analysis. Clin Nucl Med 40: 774-779, 2015.

12. Rivkees SA: Controversies in the management of Graves' disease in children. J Endocrinol Invest 39: 1247-1257, 2016.

13. Ma C, Xie J, Wang H, Li J and Chen S: Radioiodine therapy versus antithyroid medications for Graves' disease. Cochrane Database Syst Rev 2: CD010094, 2016.

14. Ross DS, Burch HB, Cooper DS, Greenlee MC, Laurberg P, Maia AL, Rivkees SA, Samuels M, Sosa JA, Stan MN, et al: 2016 American Thyroid Association Guidelines for diagnosis and management of hyperthyroidism and other causes of thyrotoxicosis. Thyroid 26: 1343-1421, 2016. 
15. Jiang Y, Hu K, Xie W, Zheng G, Sun J, Zheng Y and Huang H: Hyperthyroidism with concurrent FMS-like tyrosine kinase 3-internal tandem duplication-positive acute promyelocytic leukemia: A case report and review of the literature. Oncol Lett 7: 419-422, 2014.

16. Dong LQ, Sun XM, Xiang CF, Wu J and Yu P: Hashimoto's thyroiditis and papillary carcinoma in an adolescent girl: A case report. Mol Clin Oncol 5: 129-131, 2016.

17. Wang LQ, Wang TY, Sun QL and Qie YQ: Correlation between thyroglobulin gene polymorphisms and autoimmune thyroid disease. Mol Med Rep 12: 4469-4475, 2015.

18. Nakabayashi K and Shirasawa S: Recent advances in the association studies of autoimmune thyroid disease and the functional characterization of AITD-related transcription factor ZFAT. Nihon Rinsho Meneki Gakkai Kaishi 33: 66-72, 2010 (In Japanese).

19. M'Rabet-Bensalah K, Aubert CE, Coslovsky M, Collet TH, Baumgartner C, den Elzen WP, Luben R, Angelillo-Scherrer A, Aujesky D, Khaw KT, et al: Thyroid dysfunction and anaemia in a large population-based study. Clin Endocrinol (Oxf) 84: 627-631, 2016
20. Fischli S, von Wyl V, Trummler M, Konrad D, Wueest S, Ruefer A, Heering K, Streuli R, Steuer C, Bernasconi L, et al: Iron metabolism in patients with Graves' hyperthyroidism. Clin Endocrinol (Oxf) 87: 609-616, 2017.

21. Szczepanek-Parulska E, Hernik A and Ruchała M: Anemia in thyroid diseases. Pol Arch Intern Med 127: 352-360, 2017.

22. Yu X, Wang Y, Wang P, Ji CH, Miao CD and Zheng S: Primary thyroid paraganglioma mimicking medullary thyroid carcinoma: A case report. Oncol Lett 10: 1000-1002, 2015.

23. Yang L, Shen X, Yan S, Yuan X, Lu J and Wei W: HbA1c in the diagnosis of diabetes and abnormal glucose tolerance in patients with Graves' hyperthyroidism. Diabetes Res Clin Pract 101: 28-34, 2013.

(i) () $($ This work is licensed under a Creative Commons International (CC BY-NC-ND 4.0) License. 